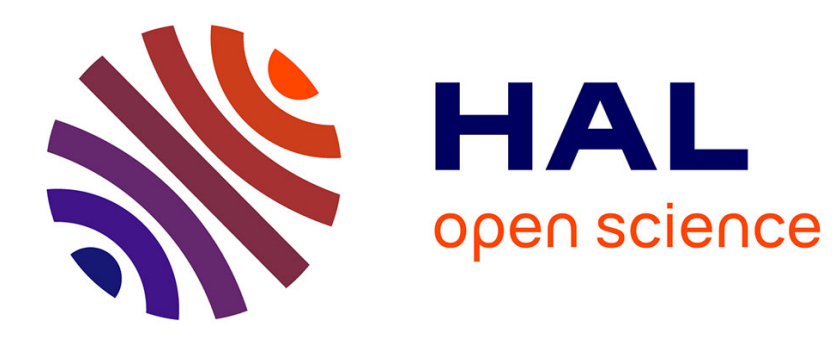

\title{
Dislocation pattern formation in metals
}

J. Kratochvil

\section{To cite this version:}

J. Kratochvil. Dislocation pattern formation in metals. Revue de Physique Appliquée, 1988, 23 (4), pp.419-429. 10.1051/rphysap:01988002304041900 . jpa-00245790

\section{HAL Id: jpa-00245790 https://hal.science/jpa-00245790}

Submitted on 1 Jan 1988

HAL is a multi-disciplinary open access archive for the deposit and dissemination of scientific research documents, whether they are published or not. The documents may come from teaching and research institutions in France or abroad, or from public or private research centers.
L'archive ouverte pluridisciplinaire HAL, est destinée au dépôt et à la diffusion de documents scientifiques de niveau recherche, publiés ou non, émanant des établissements d'enseignement et de recherche français ou étrangers, des laboratoires publics ou privés. 


\title{
Dislocation pattern formation in metals
}

\author{
J. Kratochvil
}

\author{
University of Kentucky, Lexington, KY, 40506-0046, U.S.A. \\ (Reçu le 26 mai 1987, accepté le 2 juillet 1987)
}

Résumé. - La distribution homogène des dislocations dans les métaux déformés ne reste pas stable à cause de l'agglomération des dipoles de dislocations. La formation des amas de dislocations peut être expliquée par le transfert de dipoles aux places où la concentration de contraintes produite par le glissement de dislocations sous la contrainte appliquée est importante. L'analyse linéaire de la stabilité montre que le modèle proposé permet d'écrire les plus importants traits du développement de la structure de dislocation observée pendant la déformation des cristaux de métaux par un glissement simple de la charge soit en traction ou en contraction soit alternée.

\begin{abstract}
A homogeneous dislocation distribution in deformed metals is unstable with respect to clustering of dislocation dipoles. The main reason for formation of the dislocation clusters is a net dipole drift to stress concentrations created by glide dislocations in a applied stress state. Linear stability analysis indicates that the suggested model gives a reasonable description of the most important features of the early stage of dislocation structure development as observed in metal crystals in single slip under both unidirectional and cyclic deformation.
\end{abstract}

1. Introduction.

Dislocations produced in a deformed metal tend to cluster into various kinds of dense formations separated by relatively dislocation free regions. This phenomenon which underlies such important effects as work hardening and localization of plastic deformation is very common. It occurs in single crystals as well as in polycrystais, both in pure metals and in alloys.

Typically, at the onset of plastic deformation a relatively uniform distribution of dislocations is observed. As the deformation proceeds this initial distribution becomes unstable; the dislocations start to aggregate into clusters which tend to form a three-dimensional pattern. As deformation increases this pattern may loose stability again and undergo a further transition into an even more complex restabilized state. The well known stages of tensile or cyclic strain hardening of metal crystals, each of them connected with a characteristic dislocation pattern, probably may be treated as a consequence of such transitions. The effects seem to be similar to the structuralization of other nonlinear systems driven sufficiently far from thermodynamic equilibrium. Mosaic-like patterns have been observed in number of physical, chemical and biological systems (e.g. Benard's cells in fluids, lamelar structures formed during solidification, patterns in chemically reacting systems; spinodally decomposed alloys, etc.).

Several attempts have been made to find a reason for dislocation pattern formation [1-9]. In the most recent theory Walgraef and Aifantis treated

[1] On leave from the Institute of Physics, Czechoslovakia Academy of Sziences, 18040 Prague, Czechoslovakia. the dislocation population in a stressed metal crystal as a reaction-diffusion system [4-7]. The main driving force for dislocation clustering is seen as a reduction of strain energy by a suitable rearrangement of dislocations. See, for example, the description by Kuhlmann-Wilsdorf [8,9] and Holt [1]. The present model of an initial stage of dislocation pattern formation is based on a more detailed mechanism of dislocation clustering which was suggested in [10] and is formulated in mathematical terms in this and two other publications. ${ }^{2}$

The problem to be addressed is the loss of stability of initially uniform dislocation distribution and the first stage of clustering as occurs in cubic metal crystals during tensile or cyclic deformation. In [10] it was proposed that the first stage of structuralization is governed by the behaviour of edge dislocation dipoles formed during, the deformation process. The leading mechanism is a net drift of dipoles to positions of stress concentration. Such concentrations can be created by glide dislocations stuck at the already formed clusters of dipoles when an applied stress is imposed. Due to this effect a fluctuation in dipole density can serve as an embryonic cluster from which a dislocation pattern may develop.

\section{Summary of Dislocation Structure Observations.}

The main motivation for the suggested model of pattern formation was the extensive experimental study of dislocation structure development in $\mathrm{Fe}-$ Si crystals deformed in tension, compression [11,

[2] R. Kalus: Master degree thesis, Charles University, Prague 1987, R. Kalus, J. Kratochvíl and S. Libovický: On Geomeiry of Dislocation Patterns in Metals (ir prenaration). 
12] and cycling [13, 14, 15] in single slip modes. Similar dislocation behaviour is observed in pure f.c.c. metal crystals oriented for single slip $[16,17]$.

Figures 1 and 2 show examples of the disloca- tion patterns developed during tensile and cyclic deformation, respectively, observed on (I01) sections (a) parallel to the primary slip plane and (101) sections (b) normal to it (see the insert in Fig. 1). In these typical micrographs the primary dislocations are aggregated into

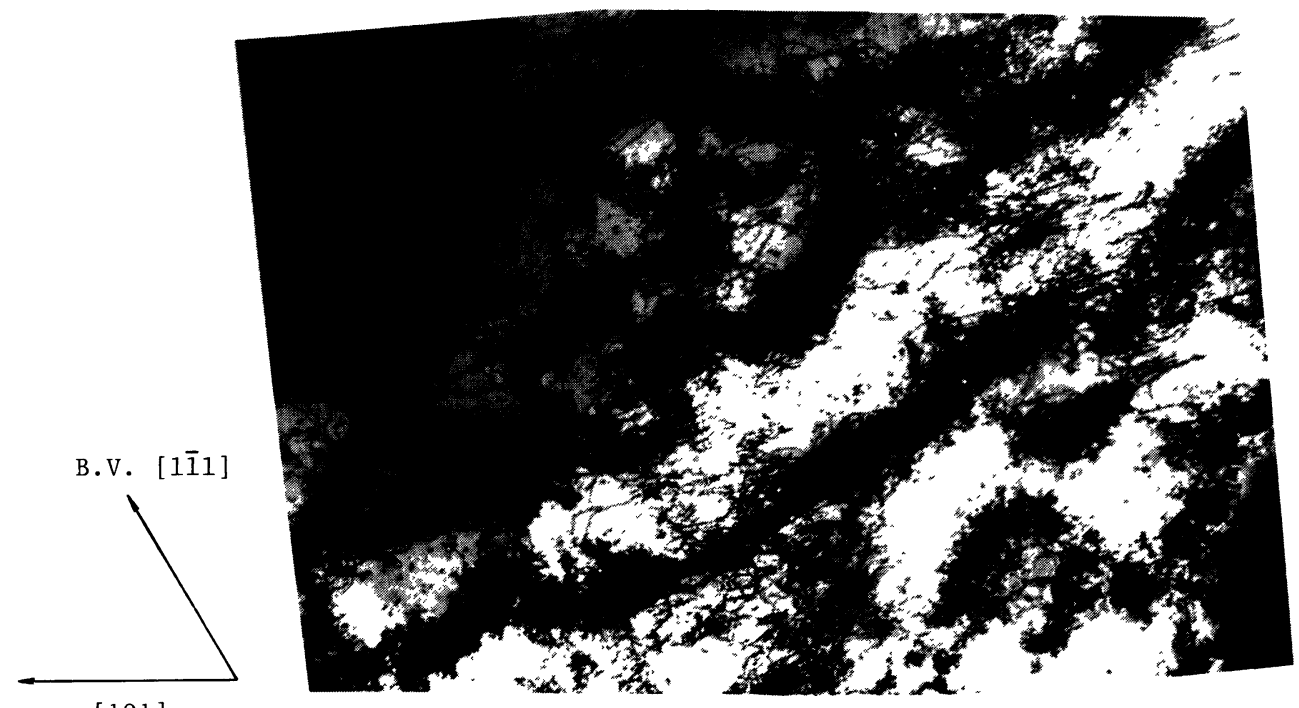

[101]

a) A section parallel to the glide plane ( $\overline{1} 01$ )
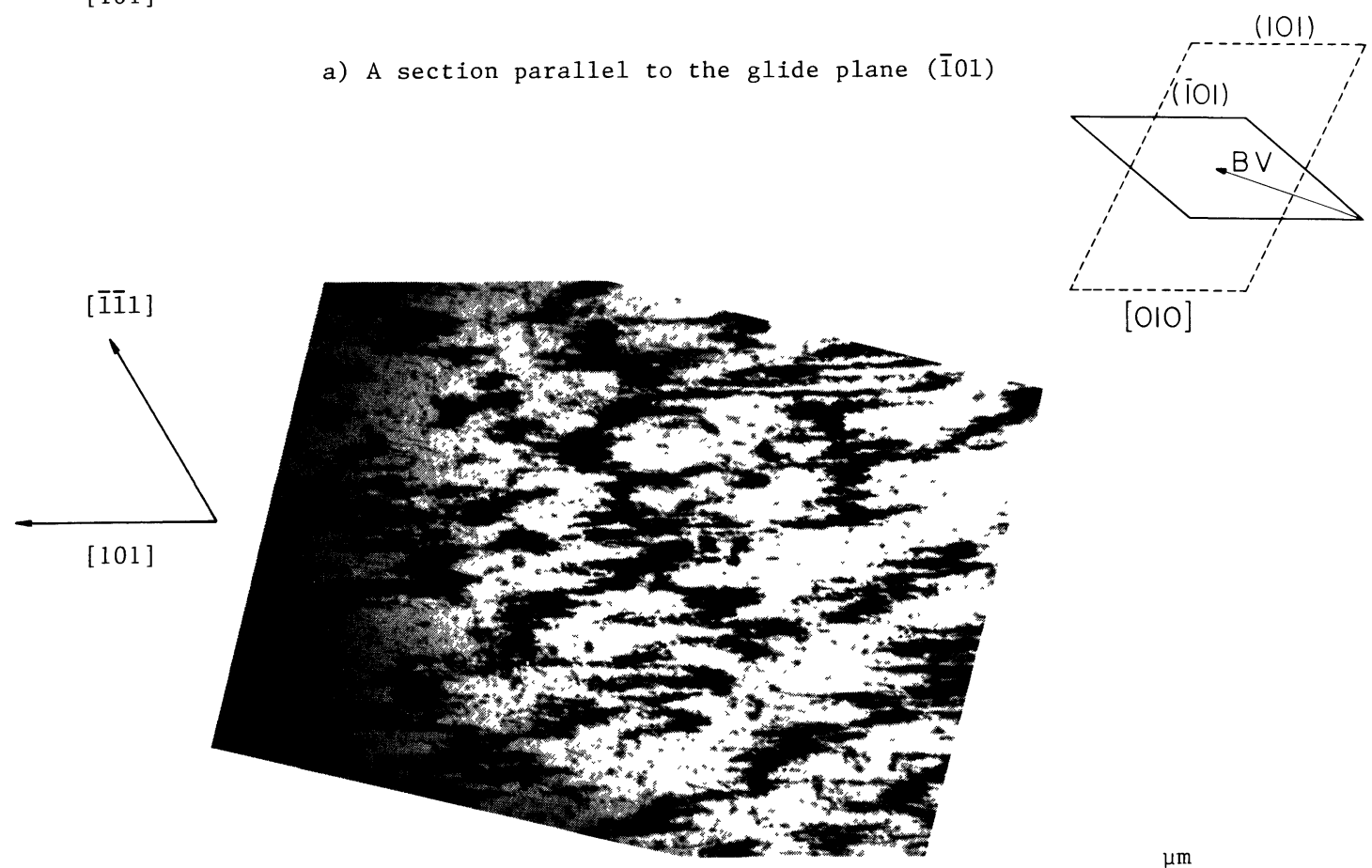

[101]

b) A section parallel to the secondary plane (101)

Fig. 1. TEM micrographs of the dislocation structure of Fe+0.9\%Si single crystal, plastic shear strain 0.45 . Courtesy of S. Libovický. 
CYCLING

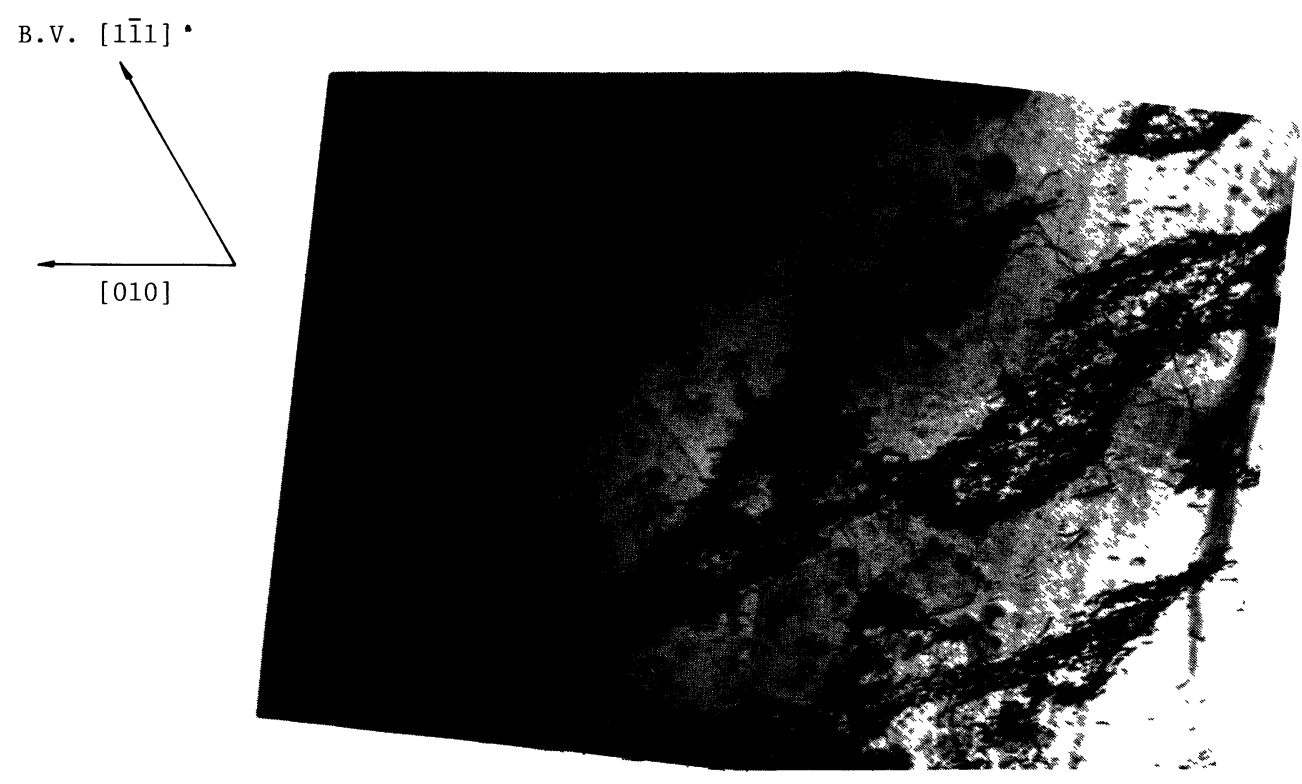

a) A section parallel to the glide plane (ī01)

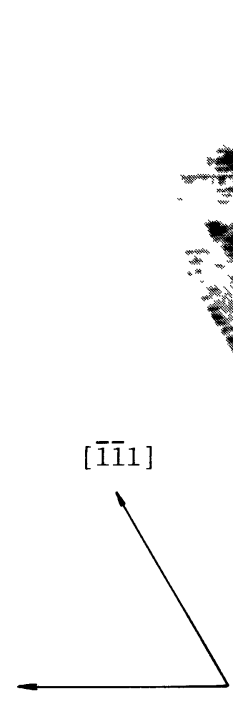

[010]

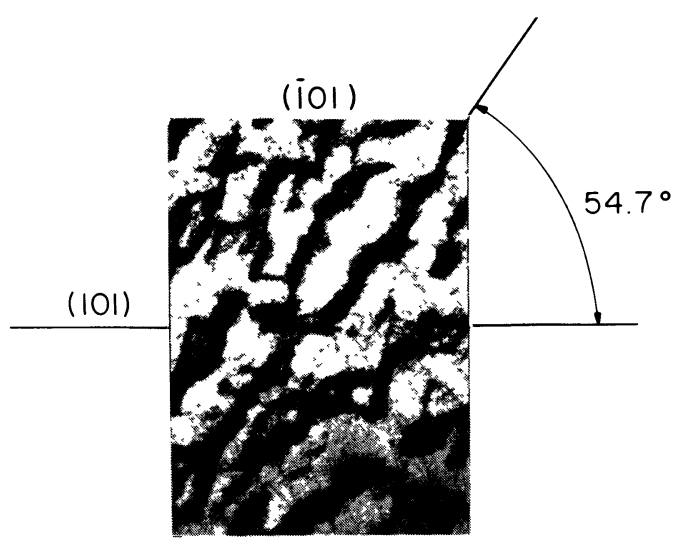

c)

b) A section paralle1 to the secondary plane (101)

Fig. 2. TEM micrographs of the dislocation structure of Fe+0.9\%Si single crystal, plastic strain amplitude $2 \times 10^{-3}, 400$ cycles. Insert c): A section parallel to the glide plane (101), Fe+3\%Si single crystal after cycling, same magnification as in cases a) and b). Courtesy of S. Libovický. 
clusters called braids (bundles) in the case of tension, Fig. 1, and veins (loop patches) for cycling, Fig. 2. Dislocations of approximately edge character in the form of extended prismatic loops dominate the clusters. The dislocation density in the regions between clusters (channels) remains low during the deformation.

As documented by Figs. 1 and 2 in the first stage of clustering there is no principal difference between the dislocation structure developed during unidirectional deformation and cycling. The overall geometry of the patterns, the preferred crystallographic orientation and the size of the clusters and channels are about the same. However, the clusters are more sharply defined after cycling than after tension or compression. In addition, there is a higher dislocation density in the channels after tension. It is, however, remarkable that the size of the pattern dimension decreases rapidly with increasing silicon content [15]. This can be observed by comparing Fig. $2 \mathrm{a}$ which represents $\mathrm{Fe}+0.9 \% \mathrm{Si}$ and the micrograph in the insert in Fig. 2, which is $\mathrm{Fe}+3 \% \mathrm{Si}$, in both cases the magnification is the same.

The clusters viewed on the (ī01) plane (Figs. $1 \mathrm{a}, 2 \mathrm{a}$ and the insert in Fig. 2) show a tendency to be oriented approximately along [111] closepacked direction in the ( $\overline{1} 01)$ slip plane which is inclined about $70^{\circ}$ to the Burgers vector [1i1]. On the (101) sections, Figs. $1 \mathrm{~b}$ and $2 \mathrm{~b}$, which intersect the clusters at an angle of about $55^{\circ}$ (see the insert Fig. 2) the dislocation patterns exh1bit two distinct directions each of which makes an angle somewhat less than $45^{\circ}$ to the trace of the primary slip plane. The theory described in the following sections gives an interpretation of these observations.

3. Behavior of Dipoles in an Inhomogeneous Stress Field.

The suggested mechanism of the early stage of the dislocation pattern formation was motivated by the fact that the clusters, both the braids and veins, consist primarily of dislocation dipoles. The diclocation dipoles are generated during deformation due to interactions among dislocations. The great quantity of elongated loops created behind jogged screw dislocations was reported as a dominant deformation feature $[13,18]$. Moreover, the screw parts of dislocation dipole annihilate much easier than the edge parts [19] producing dipoles of approximately edge character in form of extended prismatic loops.

In a homogeneous stress field a dipole should remain stationary because no net force is exerted on it. However, the dipole is polarized, i.e., extended or compressed, due to the forces acting on the dislocations of the dipole. Four possible stable equilibrium configurations of an edge dipole are shown in Fig. 3. In a positive shear stress the configurations called in Fig. 3 positive are extended (the distance between the dislocations increases) while the configurations called negative are compressed. A negative shear stress has the opposite effect. At a sufficiently high level of stress the larger dipoles, which are held together by smaller mutual force between the dislocations, are decomposed. This instability of dipoles, which does not seem to be crucial in the first stage of structuralization, may prove to be essential for transformations of braids or veins into sheets, walls or cells observed in the later stages of strain hardening.

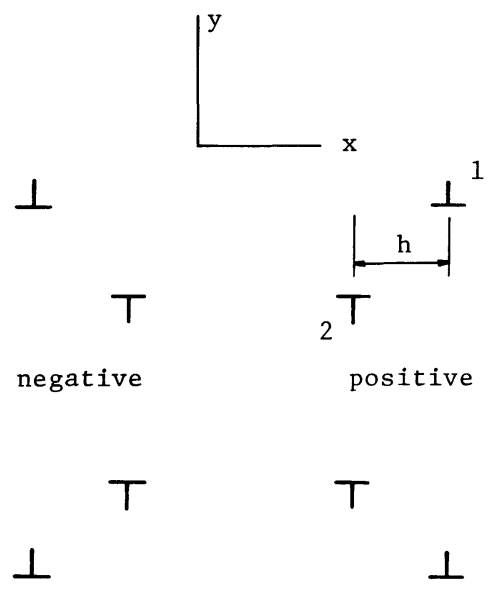

Fig. 3. Dipole configurations

The net force acting on a dipole is non-zero in a shear stress gradient and may cause glide of the dipole as a whole. As this force depends on the distance between the dislocations of the dipole, it has, due to polarization of the dipoles, an asymmetric nature; it is greater in an oxtended state than in the compressed one. It is imporiant to realize that dipoles in extended states are always forced by a stress gradient to the place of the highest absolute value of stress. On the other hand the compressed dipoles are moved in a stress gradient towards the place of the lowest absolute value of stress. Moreover, due to the asymmetry caused by the polarization the extended dipoles move "up hill" faster than the compressed ones move "down hil1".

In a symmetric cyclic loading the sign of the stress changes in each half-cycle. In this case the extended and compressed states of the dipoles alternate and in a stress gradient the dipoles move forward and backward but due to asymmetry of the forces the net drift of each dipole is in the direction of the higher absolute value of the stress, i.e. all dipoles move "up hill" to the stress concentrators. In the next sections it is shown that "up hill" drift of dipoles both in unidirectional and cyclic deformation destabilizes the uniform distribution of dislocation and initiates clustering.

\section{Equations Governing Pattern Formation.}

In the suggested model the dislocation population is idealized. It is assumed simply that it consists of mobile dislocations, which produce plastic deformation, and dislocation dipoles, which cause strain hardening. The glide dislocations enter the theory implicitely as slip strain. The slip causes an internal stress which together with the applied stress controls the drift of the dipoles.

Dipoles. In the formulation of the governing equations for dipoles keep in mind that according to the analysis of Section 3 there are two types of dipoles: positive and negative. In a given shear stress gradient the positive and negative dipoles move in opposite directions and due to the polarization effect they have different mobilities. 
Therefore each group will be considered separately. First consider the balance law for the positive dipoles: the rate of accumulation of the positive dipoles in a small volume element $\mathrm{dV}$ must be equal to the net flux $\mathrm{J}^{+}$of these dipoles in $\mathrm{dV}$ plus the net rate of generation of the positive dipoles $\mathrm{r}^{+}$ in $\mathrm{dV}$. If the distribution of the positive dipoles is described by a density function $\rho^{+}$, the balance law has the standard differential form

$$
\frac{\partial \rho^{+}}{\partial t}=-\operatorname{div} \overrightarrow{\mathrm{J}}^{+}+\mathrm{r}^{+}
$$

As dipoles are assumed to be infinitely long in the $z$ direction, the quantities $\rho^{+}, \mathrm{J}^{+}$and $\mathrm{r}^{+}$are functions of time $t$ and the coordinates $x$ and $y$.

Taking the y direction normal to the slip plane in the single slip case considered, only the $x-$ component $\mathrm{Jx}^{+}$of $\overrightarrow{\mathrm{J}}^{+}$is non-zero.

The quantities $\rho^{+}, \mathrm{J}_{\mathrm{x}}^{+}$and $\mathrm{r}^{+}$refer to the total number of positive dipoles per unit volume regardless of their size. Instead of considering a detailed size distribution it is assumed simply that all of the dipoles have the same size equal to the average size of the population. (Also for simplicity it is assumed that the average size of positive and negative dipoles is the same).

The flux $\mathrm{Jx}^{+}$can be expressed as $\mathrm{J}^{+}=\rho^{+} \mathrm{v}^{+}$, where $\mathrm{v}^{+}$means the velocity of a positive dipole $\mathrm{v}^{+}=[\mathrm{v}(1)+\mathrm{v}(2)] / 2$; where $\mathrm{v}(1)$ and $\mathrm{v}(2)$ denote the velocities of the dislocations 1 and 2 of the dipole (see Fig. 3). As clustering of dipoles is a relatively slow process, the velocities $\mathrm{v}^{+}, \mathrm{v}(1)$ and $\mathrm{v}(2)$ seem to be much lower than velocities of glide dislocations at the yield stress. According to the solution hardening theory of Labusch's et. al. [20-22], at very low dislocation velocities, a linear dependence is expected between velocity $v$ and the resolved shear stress $\sigma_{x y}$. In that case we have

$$
\begin{gathered}
\mathrm{v}(1)=\mathrm{B}\left[\sigma_{\mathrm{xy}}+\mathrm{d}^{+} \frac{\partial \sigma_{\mathrm{xy}}}{\partial \mathrm{x}}-\sigma_{\mathrm{o}}\right], \\
\mathrm{v}(2)=\mathrm{B}\left[-\sigma_{\mathrm{xy}}+\sigma_{0}\right],
\end{gathered}
$$

where $\sigma_{x y}$ is the shear stress in the slip plane, $\mathrm{d}^{+}$represents the distance between the dislocations 1 and 2 measured in the slip plane and $\sigma_{0}$ means stress between the dislocations. In the theory of Labusch et a1. [20] the parameter B depends on elastic constants, solution concentration and temperature. Using (2) the flux $\mathrm{J}_{\mathrm{x}}^{+}=$ $\rho^{+}[\mathrm{v}(1)+\mathrm{v}(2)] / 2$ is

$$
\mathrm{J}_{\mathrm{x}}^{+}=\frac{\mathrm{B}}{2} \rho^{+} \mathrm{d}^{+} \frac{\partial \sigma_{\mathrm{xy}}}{\partial \mathrm{x}}
$$

Note that if instead of Labusch's et al. theory some other dependence of the dislocation velocity on stress is considered (e.g. a power law $v^{\sim}\left(\sigma_{x y}\right)^{n}$, $\mathrm{n}$ is a constant), the dependence of $\mathrm{J}_{\mathrm{x}}^{+}$on stress gradient in Eq. (3) need not be linear. However, due to the linearization employed in standard stability analyses (Section 5) the only substantional change is a modified analytical form of the diffusion coefficient $D$ in (28).

In a similar way we obtain for the flux $\mathrm{J}_{\mathrm{x}}{ }^{-}$of the negative dipoles of density $\rho^{-}$

$$
\mathrm{J}_{\mathrm{x}}{ }^{-}=-\frac{\mathrm{B}}{2} \rho^{-} \mathrm{d}^{-} \frac{\partial \sigma_{\mathrm{xy}}}{\partial \mathrm{x}}
$$

Due to the polarization of the dipoles the distances $\mathrm{d}^{+}$and $\mathrm{d}^{-}$are not the same; for small changes of the equilibrium distance $h$ between the dislocations due to the stress field $\sigma_{x y}$ we get

$$
\begin{aligned}
& d^{+}=h\left[1+q \sigma_{x y}\right], \\
& d^{-}=h\left[1-q \sigma_{x y}\right],
\end{aligned}
$$

where $q=4 \pi(1-v) h / G b$ which can be easily derived from the force relation between two parallel edge dislocations of opposite signs and Burgers vectors $b$ in an isotropic elastic continuum of shear modulus $G$ and Poissons ratio $\nu$. To give an example: in $\mathrm{Fe}+0.5 \% \mathrm{Si}\left(\mathrm{G}=8 \times 10^{4} \mathrm{MPa}\right.$, $v=1 / 3, b=0.24 \mathrm{~nm}$ ) at a stress level of $35 \mathrm{MPa}$, the difference $d^{+}-d^{-}$for a dipole of a typical size $\mathrm{h}=10 \mathrm{~nm}$ is $30 \%$.

The net generation of dipoles is a result of complicated interactions among dislocations and encounters of glide dislocations with existing dipoles. However, for low $\rho=\rho^{+}+\rho^{-}$the controlling mechanism seems to be the rate of encounters of the glide dislocations which is proportional to the rate of slip p. Therefore it is assumed that the rate of the generation of positive $r^{+}$(or negative $r^{-}$) dipoles at low $\rho$ is given by

$$
\mathrm{r}^{ \pm}=\mathrm{A}^{ \pm}\left|\frac{\partial \mathrm{p}}{\partial \mathrm{t}}\right|
$$

Using (3)-(6) in (1) we get the balance law for positive (+) and negative (-) dipoles in the form

$$
\frac{\partial \rho^{ \pm}}{\partial t}=-\frac{\partial}{\partial x}\left[D^{ \pm} \frac{\partial \sigma_{x y}}{\partial x}\right]+A^{ \pm}\left|\frac{\partial p}{\partial t}\right|
$$

where

$$
\mathrm{D}^{ \pm}= \pm \frac{\mathrm{B}}{2} \rho^{ \pm} \mathrm{h}\left[1 \pm \mathrm{q} \sigma_{\mathrm{xy}}\right]
$$

For higher values of dipole density $\rho=\rho^{+}+\rho^{-}$it would be necessary to consider that $\mathrm{D}^{ \pm}$and $\mathrm{A}^{ \pm}$ depend in more complicated ways (see Section 7 ) on $\rho, \rho^{+}, \rho^{-}$.

Slip Strain, Non-Local Hardening. The slip strain rate $\partial \mathrm{p} / \partial t$ and shear stress $\sigma_{x y}$ in (8) can be related by

$$
\frac{\partial p}{\partial t}=C\left|\frac{\sigma_{x y}}{\tau}\right|^{m-1} \frac{\sigma_{x y}}{\tau},
$$

where $\mathrm{C}$ depends on glide dislocation density and $\mathrm{m}$ is a material constant. The quantity $\tau>0$ means the flow stress. However, the que itative analysis of this article is independent of the particular choice of the form of (9).

The flow stress increases due to strain hardening. According to the simplifying assumption introduced at the beginning of this Section the growing density of dipoles is responsible for strain hardening. However, to assume that the flow 
stress is just a function of the dipole density $\rho=\rho^{+}+\rho^{-}$is not sufficient for the present consideration which is intended to describe a collective dislocation behavior on a scale of the order of one micron. For instance a shortening of dislocation sources due to narrowing of the channels between clusters contributes to strain hardening as well. Thus, the dipoles in clusters not only harden the clusters but also the relatively dislocation free regions of the crystal between them. The non-local interaction which incorproates such effects is described in a phenomenological way. The flow stress is assumed to be of the form

$$
\tau(x, y, t)=\tau_{0}+\int_{-\infty}^{\infty} M\left(x-x^{\prime}\right) \rho\left(x^{\prime}, y, t\right) d x^{\prime},
$$

where $\tau$ is the yield stress. Relationship (10) states that the dipole density $\rho$ at a point $x^{\prime}$ influences the flow stress at other points of the same slip plane. As all dipoles participate in hardening, the total density $\rho=\rho^{+}+\rho^{-}$is employed in (10). A generalization of (10) would be to incorporate a non-local effect in the y dires-tion as well.

To give a possible interpretation of (10) consider a primitive model of the dislocation source shortening effect. A source centered at $\mathrm{x}$ would ordinarily operate at a stress inversely proportional to its size. However, the presence of a dipole at $x^{\prime}$ such that $2\left|x-x^{\prime}\right|$ is smaller than the source size will interfere with the operation of the source. In fact, the dipole will force the source asymmetrically towards $x$ and increase the stress required for it to operate. A higher density $\rho$ at $x$ ' may block more of such sources. Hence, the increase in the average stress needed to operate shorter sources represented by the integrand in (10) is identified with the increase of the flow stress at $x$ caused by the dipole density $\rho$ at $x^{\prime}$. The total increase of $\tau$ is the sum of these contributions. As a higher stress is needed to operate smaller sources we can expect that $M$ is a positive, symmetric function decreasing with increasing $\left(x-x^{\prime}\right)$ and approaching zero at infinity. As dipoles block sources mainly on their own slip plane, $M$ in (10) is approximately independent of the y coordinate. Its form is related to the size distribution of the sources and should be derivable from detailed statistics of the source shortening effect.

Stress Field. The stress which appears in (7)(9) must satisfy the conditions of equilibrium, compatibility and Hookes law. In agreement with the assumed infinite length of the dipoles in the $z$ direction the model is treated as a plane strain problem. For simplicity elastic isotropy is adopted. Then the strain $\underset{\sim}{\varepsilon}=\underset{\sim}{\varepsilon}(x, y, t)$ has three known components: $\varepsilon_{\mathrm{zz}}=\varepsilon_{\mathrm{xz}}=\varepsilon_{\mathrm{yz}}=0$. The stress $\stackrel{\sigma}{\sigma}=\sigma(\mathrm{x}, \mathrm{y}, \mathrm{t})$ has two: $\sigma_{\mathrm{xz}}=\sigma_{\mathrm{yz}} \stackrel{\mathrm{yz}}{=} 0$. The re-maining components of the stress and strain are governed by the following equations.

Equilibrium conditions:

$$
\begin{aligned}
& \frac{\partial \sigma_{x x}}{\partial x}+\frac{\partial \sigma_{x y}}{\partial y}=0, \\
& \frac{\partial \sigma_{x y}}{\partial x}+\frac{\partial \sigma_{y y}}{\partial y}=0,
\end{aligned}
$$

Compatibility condition:

$$
\frac{\partial^{2} \varepsilon}{\partial y^{2}}+\frac{\partial^{2} \varepsilon}{\partial x^{2}}=2 \frac{\partial^{2} \varepsilon}{\partial x \partial y},
$$

Hooke law:

$$
\begin{aligned}
& \varepsilon_{x x}=\frac{1}{E}\left[\sigma_{x x}-\nu\left(\sigma_{y y}+\sigma_{z z}\right)\right], \\
& \varepsilon_{y y}=\frac{1}{E}\left[\sigma_{y y}-\nu\left(\sigma_{x x}+\sigma_{z z}\right)\right], \\
& \varepsilon_{z z}=0=\frac{1}{E}\left[\sigma_{z z}-\nu\left(\sigma_{x x}+\sigma_{y y}\right)\right], \\
& \varepsilon_{x y}=\frac{\sigma_{x y}}{2 G}+p,
\end{aligned}
$$

where $E=2 G(1+\nu)$ is Young modulus. In the case of cycling $\varepsilon$ and $\mathrm{p}$ mean the strain and plastic strain in a half cycle, respectively.

\section{Stability}

The set of equations (7)-(14) represents the system for eleven unknown quantities $\rho^{+}, \rho^{-}, \sigma_{\mathrm{xx}}$ $\sigma_{y y}, \sigma_{z z} \cdot \sigma_{x y}, \varepsilon_{x x}, \varepsilon_{y y}, \varepsilon_{x y}, \tau$ and $p$. The conditions under which a homogeneous solution of this system becomes unstable due to an infinitesimal perturbation can be determined by standard methorls.

Homogeneous Solution. From (7)-(14) it follows that a spatially uniform solution in an infinite crystal under strain controlled conditions with only one non-zero component, $\bar{\varepsilon}_{\mathrm{xy}} \neq 0$, and the initial conditions $\bar{\sigma}_{x y}=\tau_{0}, \bar{\rho}^{+}(0)=0, \bar{\rho}^{-}(0)=0$, $\overline{\mathrm{p}}(0)=0$ satisfy the equations (homogeneous solution is denoted by a superposed bar)

$$
\begin{gathered}
\frac{d \bar{\rho}}{d t}=A^{+} C\left|\frac{\bar{\sigma}_{x y}}{\tau_{0}+H_{o} \bar{\rho}}\right|^{m}, \\
\frac{d \bar{\rho}^{-}}{d t}=A^{-} C\left|\frac{\bar{\sigma}_{x y}}{\tau_{o}+H_{o} \bar{\rho}}\right|^{m}, \\
\frac{d \bar{\sigma}_{x y}}{d t}=2 G\left[\frac{d \bar{\varepsilon}_{x y}}{d t}-\frac{C}{\left(\tau_{o}+H_{o} \bar{\rho}\right)^{m}}\left|\bar{\sigma}_{x y}\right|^{m-1} \bar{\sigma}_{x y}\right], \\
H_{o}=\int M(x) d x,
\end{gathered}
$$

and the variables $\sigma_{\mathrm{xx}}, \sigma_{\mathrm{yy}}, \sigma_{\mathrm{zz}}, \varepsilon_{\mathrm{xx}}, \varepsilon_{\mathrm{yy}}$ are equal to zero. The equation governing the total dipole density $\bar{\rho}=\bar{\rho}^{+}+\bar{\rho}^{-}$follows from (15)

$$
\frac{\mathrm{d} \bar{\rho}}{\mathrm{dt}}=\mathrm{AC}\left|\frac{\bar{\sigma}_{\mathrm{xy}}}{\tau_{\mathrm{o}}+\mathrm{H}_{\mathrm{o}} \bar{\rho}}\right|^{\mathrm{m}},
$$

where $\mathrm{A}=\mathrm{A}^{+}+\mathrm{A}^{-}$. The densities $\bar{\rho}^{+}$and $\bar{\rho}^{-}$can be expressed through $\bar{\rho}$ as 


$$
\bar{\rho}^{+}=\frac{A^{+} \bar{\rho}}{A}, \quad \bar{\rho}^{-}=\frac{A^{-} \bar{\rho}}{A} .
$$

Linear Stability. To investigate the linear stability of the above solution one is looking for the fastest growing perturbation in the form of an infinitesimal wave. The perturbed solution is assumed in the same form for all variables $\xi=\bar{\xi}+\hat{\xi} \exp \left(i k_{x} x+i k_{y} y+\omega t\right)$, where $\xi$ means any of the considered variables, $\bar{\xi}$ is the homogeneous solution, $\hat{\xi}$ is the infinitesimal amplitude of the corresponding perturbation, $\mathrm{k}_{\mathrm{x}}$ and $\mathrm{k}_{\mathrm{y}}$ are the components of the wave vector $\vec{k}$ in the $\mathrm{x}$ and $\mathrm{y}$ directions, and $\omega$ is the amplification factor which determines the rate of growth or decay of the perturbation. If we use the perturbed solution in the system (7)-(14), employ (15)-(19) and linearize the resulting system of equations, we obtain for the amplitudes $\hat{\rho}, \hat{\varepsilon}_{x y}, \hat{\varepsilon}_{x x}, \hat{\varepsilon}_{y y}, \hat{\sigma}_{y y}$, $\hat{\sigma}_{x x}, \hat{\sigma}_{y y}$

$$
\begin{gathered}
\omega \hat{\rho}=D k_{x}{ }^{2}\left|\hat{\sigma}_{x y}\right|+\operatorname{AN}\left[\left|\hat{\sigma}_{x y}\right|-\tilde{\mathrm{H}} \hat{\rho}\right] \\
\omega \hat{\sigma}_{x y}=2 \mathrm{G}\left\{\omega \hat{\varepsilon}_{\mathrm{xy}}-\mathrm{N}\left[\hat{\sigma}_{\mathrm{xy}} \mp \tilde{\mathrm{H}} \hat{\rho}\right]\right\} \\
\mathrm{k}_{\mathrm{x}} \hat{\sigma}_{\mathrm{x} x}+\mathrm{k}_{\mathrm{y}} \hat{\sigma}_{\mathrm{x} y}=0, \quad \mathrm{k}_{\mathrm{x}} \hat{\sigma}_{\mathrm{x} y}+\mathrm{k}_{\mathrm{y}} \hat{\sigma}_{\mathrm{yy}}=0, \\
\mathrm{k}_{\mathrm{y}}{ }^{2} \hat{\varepsilon}_{\mathrm{xx}}+\mathrm{k}_{\mathrm{x}}{ }^{2} \hat{\varepsilon}_{\mathrm{yy}}=2 \mathrm{k}_{\mathrm{x}} \mathrm{k}_{\mathrm{y}} \hat{\varepsilon}_{\mathrm{x} y}, \\
\hat{\varepsilon}_{\mathrm{xx}}=\mathrm{a}_{1} \hat{\sigma}_{\mathrm{xx}}-\mathrm{a}_{2} \hat{\sigma}_{\mathrm{yy}}, \quad \hat{\varepsilon}_{\mathrm{yy}}=-\mathrm{a}_{2} \hat{\sigma}_{\mathrm{x} x}+\mathrm{a}_{1} \hat{\sigma}_{\mathrm{yy}},
\end{gathered}
$$

where $a_{1}=\left(1-v^{2}\right) / E, a_{2}=v(1+v) / E$ and $\tilde{H}$ is related to the Fourier transform of the function $\mathrm{M}(\mathrm{x})$ introduced in (10)

$$
\tilde{H}\left(k_{x}\right)=\frac{\left|\bar{\sigma}_{x y}\right|}{\tau} \int_{-\infty}^{\infty} M(x) \exp \left(-k_{x} x\right) d x
$$

$\mathrm{N}$ denotes

$$
N=C \frac{m}{\bar{\tau}}\left|\frac{\bar{\sigma}_{x y}}{\bar{\tau}}\right|^{m-1}
$$

and the symbol $D$ is the diffusion coefficient of the total density of dipoles

$$
D=\frac{B h}{2} \quad \bar{\rho}\left[q\left|\bar{\sigma}_{x y}\right| \pm \frac{A^{+}-A^{-}}{A}\right]
$$

The upper (1ower) sign in (21) and (28) comes from the absolute values used in (7), (20), and (26) and is valid for positive (negative) shear; $\bar{\tau}=\tau_{0}+H_{0} \bar{\rho}$, and $\bar{\sigma}_{x y}, \bar{\rho}$ are determined by (16) (18)
For the amplification factor $\omega$, Eqs. (20)-(28) yield the key relation of the linear stability analysis (on1y $w$ which may exhibit instability are considered)

$$
\begin{gathered}
\omega\left(k_{x}, k_{y}\right)= \\
\frac{\sqrt{N^{2}\left[1+\alpha A \tilde{H}\left(k_{x}\right)\right]^{2}+4 \alpha D k_{x}^{2} \tilde{H}\left(k_{x}\right)}-N\left[1+\alpha A H\left(k_{x}\right)\right.}{2 \alpha}
\end{gathered}
$$

where

$$
\alpha=\frac{1-v^{2}}{E}\left[1+\frac{1}{2}\left(\frac{k_{x}^{2}}{k_{y}^{2}}+\frac{k_{y}^{2}}{k_{y}^{2}}\right)\right] .
$$

In the case of low rate sensitivity of plastic deformation, i.e. the exponent $\mathrm{m}$ in the rate equation (9) becomes high. The limit $\mathrm{m} \rightarrow \infty$ in (27) and $(29)$ reduces $\omega$ to $\omega^{*}$ given by

$$
\omega^{*}\left(k_{x}, k_{y}\right)=D \frac{k_{x}{ }^{2} \tilde{H}\left(k_{x}\right)}{1+\alpha A \tilde{H}\left(k_{x}\right)} .
$$

The amplification factor $\omega$ or $\omega^{*}$ determines the stability of a uniform distribution of dipoles. For $\omega>0$ the perturhation wave $\hat{\rho} \exp \left[i k_{x} x+\right.$ $\left.i k_{y} y+\omega t\right]$ exponentially grows and, hence, the uniform solution is unstable. For $\omega<0$ the perturbation decays and the uniform state remains stable. In the physically realistic cases, such as the example in (34), the function $M$ in (10) gives a positive value of $\tilde{\mathrm{H}}$. Then the sign of $\omega$ in (29) or (31) is determined by the sign of the diffusion coefficient $D$.

Considering the various dislocation mechanisms for the generation of dipoles [23] one may assume that $\mathrm{A}^{+}$and $\mathrm{A}^{-}$are roughly the same. Then the term $\left(\mathrm{A}^{+}-\mathrm{A}^{-}\right) / \mathrm{A}$ in $(28)$ can be neglected and, hence, $D$ is positive. Then $\omega$ given by (29) or (31) indicates instability. The uniform dipole distribution is unstable from the onset of deformation and there is a tendency to form a periodic dipole arrangement with the wavelength given by the fastest growing perturbation, i.e., the maximum $\omega$.

If at positive (negative) shear stress the production of the negative (positive) dipoles would be much higher than positive (negative), i.e. $\mathrm{A}^{-}>>\mathrm{A}^{+}\left(\mathrm{A}^{+}>>\mathrm{A}^{-}\right), \mathrm{D}$ given by (28) can be negative. Then $\omega<0$, the uniform distribution of dipoles would remain stable and there would be no tendency to clustering by the suggested mechanism. Note, however, that according to (28) even in the case that the generation of the extended dipoles is lower than that of the compressed ones D may still be positive and the minority of the extended dipoles causes instability which results in clustering of the dipole population.

\section{Dislocation Pattern}

As $\omega$ occurs in an exponential, it is convenient to ignore the growth of all waves but those near the fastest growing one. Hence, in the first approximation the wave with maximum $\omega$ determines the geometry and size of the dislocation pattern developed in the initial stage of plastic deformation. 


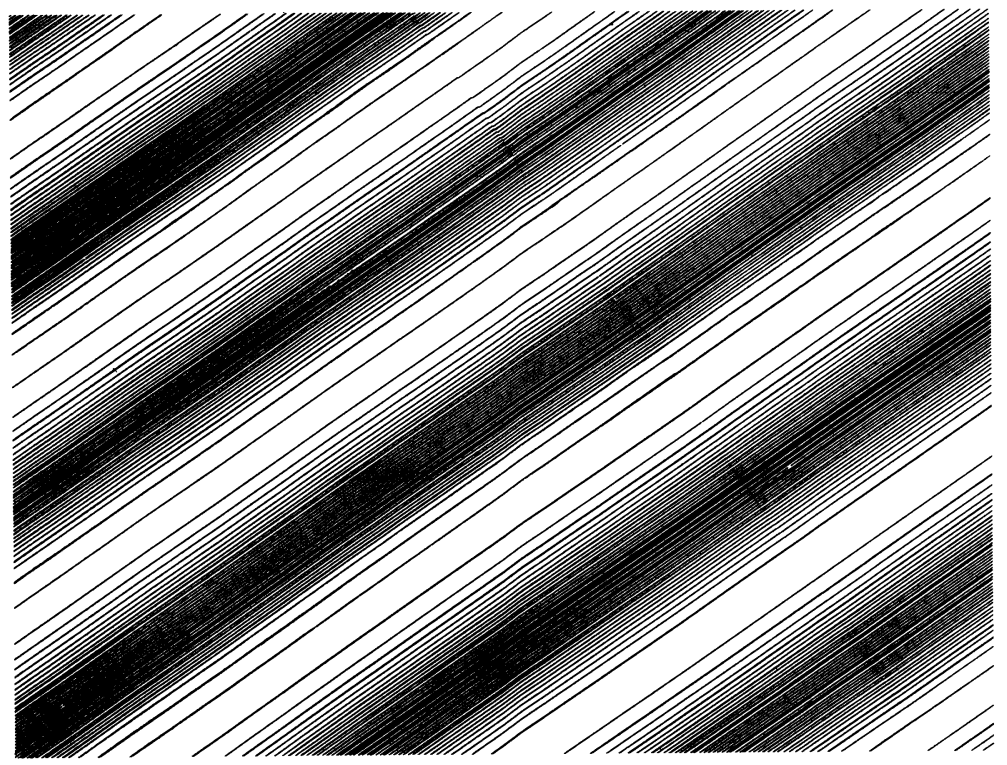

a) A section parallel to the glide plane (ī0l)

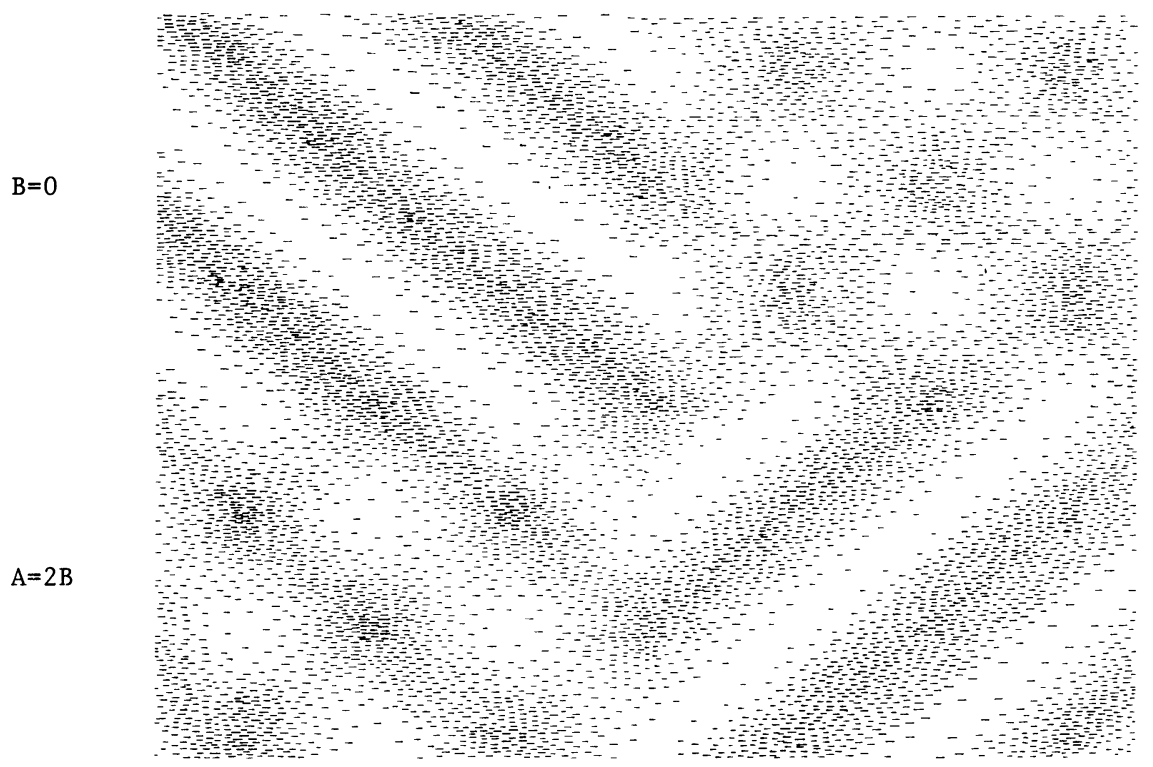

Fig. 4. Spatial distribution of dipoles according to equation (33). 
Geometry of Pattern. The amplification factor $\omega$ depends on the components $k_{x}$, $k_{y}$ of the wave vector $\vec{k}$. The component $k_{y}$ enters the relation (29) or (31) only through the factor $\alpha$ given by (30). For a positive $D$ the factor $\omega$ is maximum when $\alpha$ is minimum and equal to $2\left(1-v^{2}\right) / E$, which is reached when $k_{y}= \pm k_{x}$. Hence, the fastest growing dipole distribution wave is of the form

$$
\begin{gathered}
\rho(x, y, t)=\bar{\rho}(t)+\exp \left[\omega\left(k_{o}\right) t\right]\left\{\gamma_{1} \exp \left[i k_{o}(x+y)\right]+\right. \\
\left.\gamma_{2} \exp \left[i k_{o}(x-y)\right]\right\},
\end{gathered}
$$

where $k_{0}$ is the $k_{x}$ which maximizes $\omega$ and $\gamma_{1}, \gamma_{2}$ are complex constants. As $\rho$ must be real the spatial part $\tilde{\rho}(\mathrm{x}, \mathrm{y})$ of $(32)$ can be expressed as

$$
\tilde{\rho}(\mathrm{x}, \mathrm{y})=A \cos \mathrm{k}_{\mathrm{o}}(\mathrm{x}+\mathrm{y})+B \cos \mathrm{k}_{\mathrm{o}}(\mathrm{x}-\mathrm{y}),
$$

with $A$, B being real constants unspecified by the linear analysis.

Idealized images of the dipole distribution in a (i01) seciion parallel to the slip plane, $y=0$, and in a (101) section perpendicular to it, as predicted by (33), are shown in Fig. 4. For the (101) plot the four different cases $A=B, A=0, B=0$ and $A=2 B$ were used in the four different quadrants of the figure. This gave variety to the figure and reasonably good correspondence to the elecron micrographs in Figs. 1 and 2 . The two distinct directions that occur in the (101) section simulate we11 the experimental observations. The most obvious difference is that the observed dislocatinn patterns are much less regular. This is caused by the approximation that is used ignoring all waves except the fastest growing one. Such an approximation overemphasizes the regularity. It is important to remember that the waves with other wavelengths have also grown, although less than the fastest growing one. Irregularities and variations of the dipole density along the dipoles, as observed in Figs. $1 a$ and $2 a$, are excluded from the simulated dislocation pattern in Fig. 4, section (i01), by the assumed infinite length of the dipoles.

According to (28)-(33) the model predicts the same geometry of clusters for undirectional and cyclic deformations. This is consistent with the observation that in the first stage of deformation there is no principal difference between dislocation structures developed during tension and cycling. More sharply defined clusters after cycling than after tension and higher dislocation densities in the channels after tension can be explained by the difference in behaviours of the extended and compressed dipoles. In symmetric cycling the shear alternates and all dipoles have a net tendency to climb to the clusters. In unidirectional deformation only the extended dipoles drift "up hill". The compressed dipoles tend to occupy the lower stress channels. (According to (8) their diffusion coefficient $\mathrm{D}^{-}$is negative). There they are affected by mobile dislocations, can be dissociated into individual dislocations or transformed to tripoles, which are moved by the applied stress to the clusters. This may explain the differences between the patterns observed in tension, Fig. 1, in cycling, Fig. 2.
Size of Pattern. The size of the pattern is determined by the wavelength $\lambda=k_{o} / 2 \pi$, where $k_{o}$ maximizes $\omega$ in (29) or (31). The component $k_{\mathrm{o}_{\tilde{}}} \mathrm{de}-$ pends sensitively on the form of the function $\tilde{\mathrm{H}}\left(\mathrm{k}_{\mathrm{o}}\right)$. To give an illustrative example compatible with the model of the shortening of the dislocation sources suggested in Section 4, consider $M(x)$ in the form of a "bel1" shaped function. (See the earlier comments following Eq. (10))

$$
M(x)=\frac{M_{O}}{1+\left(\frac{x}{a}\right)^{2}},
$$

where $M_{0}$ is the height of the "be $11 "$ and a is its width at $\mathrm{M}_{\mathrm{O}} / 2$. From (34) and the Fourier transform (26) we obtain

$$
\tilde{\mathrm{H}}\left(\mathrm{k}_{\mathrm{x}}\right)=\frac{\left|\tilde{\sigma}_{\mathrm{xy}}\right|}{\tilde{\tau}} \quad M_{\mathrm{o}} \mathrm{a} \exp \left(-\mathrm{a}\left|\mathrm{k}_{\mathrm{x}}\right|\right) .
$$

For the example given by Eq. (35) the dependence of the amplification factor $\omega$ on $k_{x}$, as given by (29), and of $\omega^{*}$ on $k_{X}$, as given by (31), is shown in Fig. 5. As can be seen in this figure, the rate

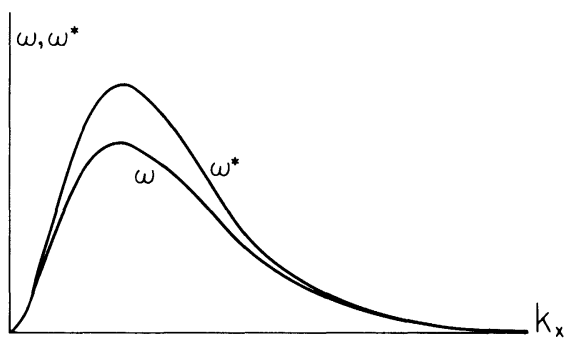

Fig. 5. Amplification factors $\omega$ and $\omega^{*}$ as $c$ function of $\mathrm{k}_{\mathrm{x}}$

sensitivity (m in (9) is small) decreases the rate of growth of the perturbation waves. The component $\mathrm{k}_{\mathrm{o}}$ of the wave vector that maximizes $\omega^{*}$ can be easily determined from (31). It satisfies the relation

$$
\mathrm{k}_{\mathrm{O}}=\frac{2 \pi\left(1-v^{2}\right)}{\mathrm{E}} \mathrm{A} \mathrm{N}_{\mathrm{O}} \exp \left(-\mathrm{ak}_{\mathrm{O}}\right)+\frac{2}{\mathrm{a}} \text {. }
$$

According to (36) the pattern size determined by $\lambda=2 \pi / k_{0}$ increases with the increasing width, $a$, decreasing dipole generation rate factor $A$, and decreasing hight $\mathrm{M}_{\mathrm{O}}$. In terms of the dislocation source shortening mechanism this means that the pattern shrinks with decreasing average size of the sources, represented in a statistical sense by a, and the increasing capability of dipoles to block their operation expressed by $\mathrm{M}_{\mathrm{O}}$.

\section{Discussion}

As indicated by the linear stability analysis the suggested model provides reasonable agreement 
with the observations summarized in Section 2. The leading wave of inhomogeneity in the dipole distribution predicts the formation of a periodic array of dislocation clusters. In a section perpendicular to the slip plane the clusters are arranged along two preferred directions oriented roughly $\pm 45^{\circ}$ to the trace of the slip plane. The electron micrographs confirm such tendency for both unidirectional (braids), Fig. 1, and cyclic (veins), Fig. 2, deformation. Within experimental scatter the observed directions agree well with the theoretical prediction, Fig. 4.

According to the model the uniform distribution of dislocations is unstable from the onset of plastic deformation. In experiments traces of clustering are observed very early, e.g., in the first half cycle [13]. However, according to (28) the diffusion coefficient $D$ which controls the rate of initial clustering is proportional to the density of dipoles $\tilde{\rho}$ which is zero initially. Therefore a slow cluster formation may be expected at the beginning of deformation.

The developed dislocation pattern is a consequence of two opposing tendencies: "up hill" drift of dipoles causing clustering and the nonlocal hardening which opposes this effect. If $\tilde{\mathrm{H}}$ is constant then $\omega$ given by (29) or (31) would be an increasing function of $\mathrm{k}_{\mathrm{x}}$ and, hence, the clustering waves with $\lambda \rightarrow 0$ would be the fastest growing instabilities. Very fine clustering is opposed by the non-local hardening represented in (29) or (31) by $\tilde{\mathrm{H}}\left(\mathrm{k}_{\mathrm{X}}\right)$. This prevents the formation of narrow channels between clusters. The size of the pattern given by the wave length $\lambda$ of the leading clustering wave is a result of a compromise between "up hill diffusion" and non-local hardening effect. As a consequence $\lambda$ depends sensitively on the form of $\tilde{\mathrm{H}}$.

On the other hand, the geometry of the pattern represented by the preferred directions seems to be independent of the plastic properties of the crystal. The reason is that the geometry is determined by the relation between the components $\mathrm{k}_{\mathrm{x}}$ and $\mathrm{k}_{\mathrm{y}}$ of the wave vector as given by the minimization of the factor $\alpha$, equation (30), which is elastic in nature. Note, however, that this is true only as long as $M$ in (10) is independent of $y$ ( $\tilde{H}$ is then a function of $k_{x}$ on $1 y$ ), i.e. non-local interaction is constrained just to the slip plane. The orientation of the preferred directions may be further influenced by elastic anisotropy through a modified form of $\alpha$.

The independence of the pattern geometry on plastic properties in the first stage of deformation seems to be in agreement with the observation that the preferred directions of the dislocation array do not vary with deformation mode, temperature, amount of deformation and solute content of alloys $[12,13]$. On the other hand, the measurements indicate $[10,12,15]$ that the size of the pattern, which is independent of the deformation mode and probably also of temperature [10], depends sensitively on the solute content. According to the results of [15] the wavelength of the pattern decreases with $\mathrm{Si}$ content $\mathrm{c}$ in $\mathrm{Fe}$ as $\lambda \sim 1 / \mathrm{c}$. In terms of the suggested model such an effect would arise if the non-local hardening were assumed to be affected by the solute content. Physically this is quite possible. An effective shortening of the dislocation sources by solute atoms would cause a smaller width of the function $M(x)$ and, as $\lambda$ decreases with decreasing $a$, the size of the pattern is reduced.
The suggested model is specified by the elastic constants, the yield stress $\tau_{0}$, the material parameters $C, m, B, h, A^{+}, A^{-}$and the function $M(x)$. The parameters $C$ and $m$ appear in the well established plastic rate equation (9) and the factor $B$ comes out of the theory of Labusch et a1. [20]. The rest of the parameters point to the less explored areas of dislocation theory. The average height of the dipoles $h$ and the coefficients $\mathrm{A}^{+}$, $\mathrm{A}^{-}$of the rate of generation of dipoles are consequences of dipole statistics. The suggested interpretation of the non-local hardening relates the function $M(x)$ in (10) to the statistics of dislocation sources and their interaction with dipole clusters. The lack of adequate microscopic understanding of the latter processes was the main reason that a simple phenomenological approach was adopted here and no attempt was made to specify values of the parameters $h, A^{+}, A^{-}$and the function $M(x)$. The emphasis was put on the qualitative nature of the problem as follows from linear stability theory.

\section{Outlook}

Any conclusion based on the model must be tempered by the fact that it is idealized in a number of respects. The linear approximations (3)-(5) were used to derive the diffusion coefficients (8) and (28) and the dipole size distribution was neglected by considering just the average size of dipoles. The balance equation (7) in its linearized form (20) is valid only at the onset of instability. Moreover, at least two other effects can modify the dislocation pattern development: saturation and sweeping effects.

If the total dipole density $\rho=\rho^{+}+\rho^{-}$reaches the order $1 / h^{2}$, the dipoles become close enough to be immobilized. Hence, one expects that the coefficient D in Eq. (20) depends on $\rho$ linearly only at small values of $\rho$. As $\rho$ becomes larger the coefficient $D$ decreases and becomes zero at the saturated value $\rho=1 / \mathrm{h}^{2}$. Analogous1ly the coefficients of the generation rates $A^{+}, A^{-}$may become dependent on $\rho, \rho^{+}, \rho^{-}$.

A dipole redistribution can be caused also by moving dislocations. Dipoles of the opposite sign to that of the shear stress are moved to lower stress regions between clusters (this is seen, if the amplitudes $\hat{\rho}^{+}$and $\hat{\rho}^{-}$are evaluated and compared) where the activity of the mobile dislocations becomes concentrated. Due to the interaction with the mobile dislocations the dipoles may be decomposed, flipped or transformed to tripoles [24] and moved away by the applied stress to the existing clusters. This sweeping mechanism contributes to the clustering process and may even become dominating in the later stages of structuralization. However, by itself it does not initiate instability of the uniform dislocation distribution. As was shown, the dipole drift mechanism does provide a destabilizing effect and may establish the basic geometry and size of the pattern.

The linear stability analysis reveals the most important features of the suggested mode1, but it overemphasizes regularity and neglects fluctuations in the pattern. A computer simulation of the pattern development based on the non-linear equations (7)-(14) might provide a more realistic picture, especially when the model is extended to cover saturation and sweeping effects.

From a broader point of view the suggested mode1 is an attempt to understand the mechanism of storage of excess dislocations in the form of 
clusters. A practically more important question is the problem of stability of these clusters. Their loss of stability may cause a local softening that triggers microscopic localization of plastic deformation. In ductile metals the localized deformation at external or internal surfaces (crack tips, grain boundaries, matrix-inclusion interfaces, etc.) may lead to crack nucleation and growth. The theoretical framework employed in this paper provides some understanding why and how the first dislocation clusters are formed. It might shed some light also on the problem of stability of these clusters.

Acknowledgments - The author is grateful to his colleagues Dr. J. Balík, Dr. A. Franěk, R. Kalus, Dr. F. Kroupa, Dr. S. Libovický and Dr. M. Saxlová from Prague and Dr. R. DeAngelis, Dr. O. W. Dillon, $\mathrm{Jr}$. and Dr. P. P. Gillis from the Unviersity of Kentucky for many helpful discussions and comments on the manuscript.

\section{References}

[1] D. J. Ho1t, J. App1. Phys. 41, 3197 (1970).

[2] V. I. Vladimirov and B. Pegel, phys. stat. sol. (b) 56, K 105 (1973).

[3] V. I. V1adimirov and A. A. Kusov, Fiz. meta1. metallovedenie 39,1150 (1975).

[4] D. Walgraef and E. C. Aifantis, J. App1. Phys. 58, 688 (1985).

[5] D. Walgraef and E. C. Aifantis, Int. J. Engn. Sci 23, 1351, 1359, 1365 (1985).

[6] E. C. Aifantis, in Mechanical Properties and Behaviour of Solids: Plastic Instabilities, (edited by C. E. Bottani and V. Balakrishnari) p. 314, World Scientific, Singapore (1986).

[7] D. Walgraef, in Mechanical Properties and Behaviour of Solids: Plastic Instabilities (edited by C. E. Bottani and V. Balakrishnari) p. 354, World Scientific, Singapore (1986).

[8] D. Kuhlmann-Wilsdorf, in Work Hardening in Tension and Fatigue, (edited by A. W. Thompson), p. 1, Am. Inst. Min. Engns., New York (1977).

[9] D. Kuhlmann-Wilsdorf, in Work Hardening (edited by J. P. Hirth and J. Weertman) p. 4, Gordon and Breach, New York (1968).

[10] J. Kratochvíl and S. Libovický, Scripta metal. 20, 1625 (1986).

[11] A. Gemperle, M. Rozsival and B. Šešták, Czech. J. Phys. B 12, 555 (1962).

[12] S. Libovický and B. Šešták, Phil. Mag. A 47, 63 (1983).

[13] M. Mori, M. Tokuwame and T. Miyazaki, Phil. Mag. A 40, 409 (1979).

[14] V. Novák, S. Libovický, B. Sešták, S. Kadečková, P. Lebduška and Z. Dlabáček, Proc. 7 th Colloquium on Mechanical Fatigue of Metals, Miskolc (Hungary) 1983, in Publications of the Technica]. University for Heavy Industry, Serie C, Vo1. 39, p. 81.

[15] S. Libovický, V. Novák and B. Šešták, Phil. Mag. (submitted).

[16] S. J. Basinski and Z. S. Basinski, in Dislocations in Solids (edited by F. R. N. Nabarro) Vol. 4, p. 261, North-Holland, Amsterdam (1979).

[17] H. Mughrabi, F. Ackermann and K. Herz, in Fatigue Mechanisms (edited by J. T. Fong) p. 69, Am. Soc. Testing and Materials, Philadelphia (1979).

[18] J. R. Low, Jr. and A. M. Turkalo, Acta metal. 10, 215 (1962).
[19] V. Essmann and H. Mughrabi, Phil. Mag. 40, 371 (1979)

[20] R. Labusch, G. Grange, J. Ahearn and P. Haasen, in Rate Processes in Plastic deformation of Materials (edited by J. C. M. Li and A. K. Mukherjee), p. 26, ASM (1975)

[21] M. Kleintges, R. Labusch, H. G. Brion and P. Haasen, Acta meta1. 25, 1247 (1977).

[22] H. Ney, R. Labusch and P. Haasen, Acta meta1. 25, 1257 (1977).

[23] J. Friede1, Dislocations, Pergamon, Oxford (1964).

[24] P. D. Neumann, Acta meta1. 19, 1233 (1971). 